\title{
Combined use of a double-lumen tube and Fogarty catheter to prevent the endobronchial spread of infection -a case report-
}

\author{
Jaewon Kim, Hyelim Lee, Han Park, and Chang-Young Jeong \\ Department of Anesthesiology and Pain Medicine, Eulji University Medical Center, Daejeon, Korea
}

Huntington's disease is a neurodegenerative disorder with an autosomal dominant inheritance pattern. Patients with Huntington's disease show an increased risk of aspiration pneumonia when the pharyngeal muscle is invaded. We report a case of advanced-stage Huntington's disease in which the patient received right middle lobectomy for a lung abscess caused by repeated aspiration. The best lung isolation technique has not yet been established in these patients. We successfully performed selective lobar isolation of the right lower and middle lobes using a double lumen tube and a Fogarty embolectomy catheter.

Key Words: Lung abscess, One-lung ventilation, Selective lobar isolation.

One of the purposes of double-lumen tube (DLT) intubation is to prevent the spread of infection to the contralateral lung [1]. However, when an abscess is present in only one pulmonary lobe and lobectomy is planned, the insertion of a DLT alone may not be sufficient to prevent the spread of infection to an ipsilateral lobe. We report selective isolation technique using a Fogarty embolectomy catheter to prevent the potential endobronchial spread of infection during surgery.

Corresponding author: Chang-Young Jeong, M.D., Ph.D.

Department of Anesthesiology and Pain Medicine, Eulji University Medical Center, 95, Dunsanseo-ro, Seo-gu, Daejeon 35233, Korea

Tel: 82-42-611-3883, Fax: 82-42-259-1111

Email: cyjeong@jnu.ac.kr

ORCID: http://orcid.org/0000-0003-2810-2511

Received: March 21, 2016.

Revised: May 3, 2016 (1st); June 7, 2016 (2nd).

Accepted: June 8, 2016.

Korean J Anesthesiol 2016 December 69(6): 619-622

https://doi.org/10.4097/kjae.2016.69.6.619

\section{Case Report}

A 48-year-old woman (height: $155 \mathrm{~cm}$, weight: $42 \mathrm{~kg}$ ) was diagnosed with Huntington's disease 15 years ago. She had a history of recurrent aspiration pneumonia induced by Huntington's disease and pulmonary tuberculosis. The patient had no history of latex allergy. We administered antibiotics and planned lobectomy of the right middle lobe to treat the abscess. Chest radiography and computed tomography (CT) findings showed a cavitary lung abscess accompanied by pneumothorax and an empyema in the right middle lobe, along with consolidation in both lower lobes.

After the patient arrived in the operating room, we conducted electrocardiography, pulse oximetry, non-invasive blood pressure monitoring, bispectral index monitoring, and preoxygenation for 3 min with the patient in a $15^{\circ}$ head-up position. General anesthesia was induced with propofol and rocuronium $(1 \mathrm{mg} / \mathrm{kg})$, and it was maintained with total intravenous anesthesia (2\% propofol and remifentanil). A mask was placed on the patient without ventilation after the drugs were administered, and intubation was performed after $30 \mathrm{~s}$. We used

(c) This is an open-access article distributed under the terms of the Creative Commons Attribution Non-Commercial License (http://creativecommons.org/ licenses/by-nc/4.0/), which permits unrestricted non-commercial use, distribution, and reproduction in any medium, provided the original work is properly cited. 

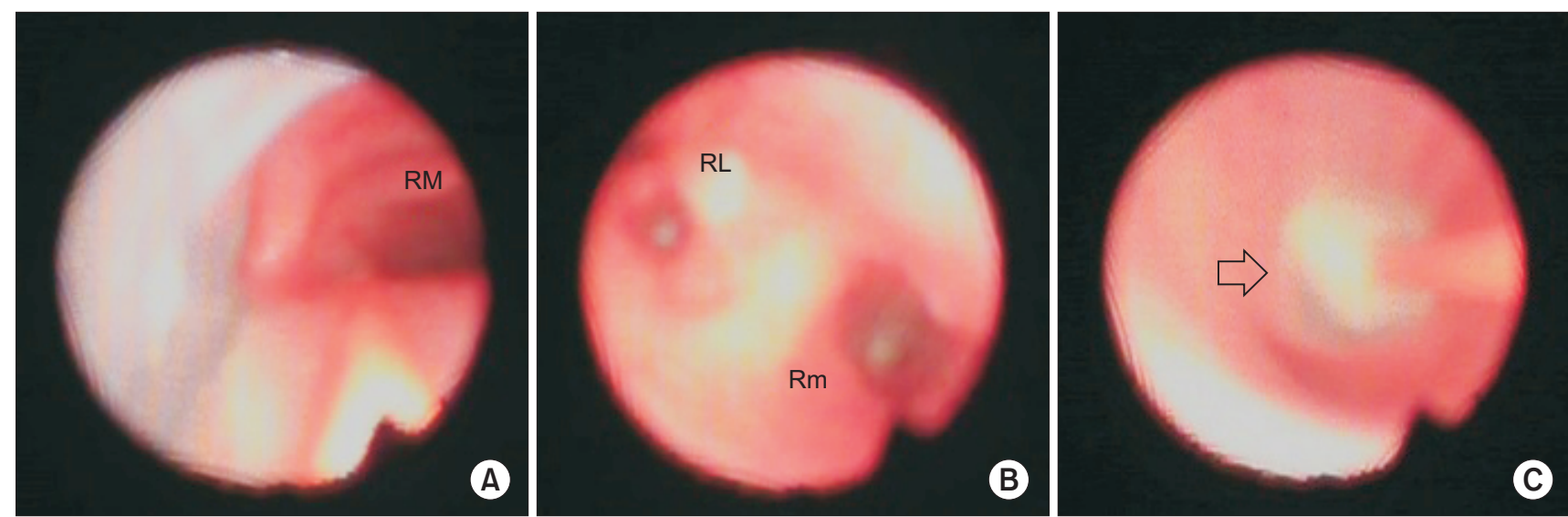

Fig. 1. (A) The catheter was inserted into the right main bronchus (RM). (B) It passed the right upper lobe, and the right lower (RL) and middle (Rm) lobes were observed. (C) Cuff ballooning (arrow) was performed at the point where the right middle lobe and right lower lobe divide.

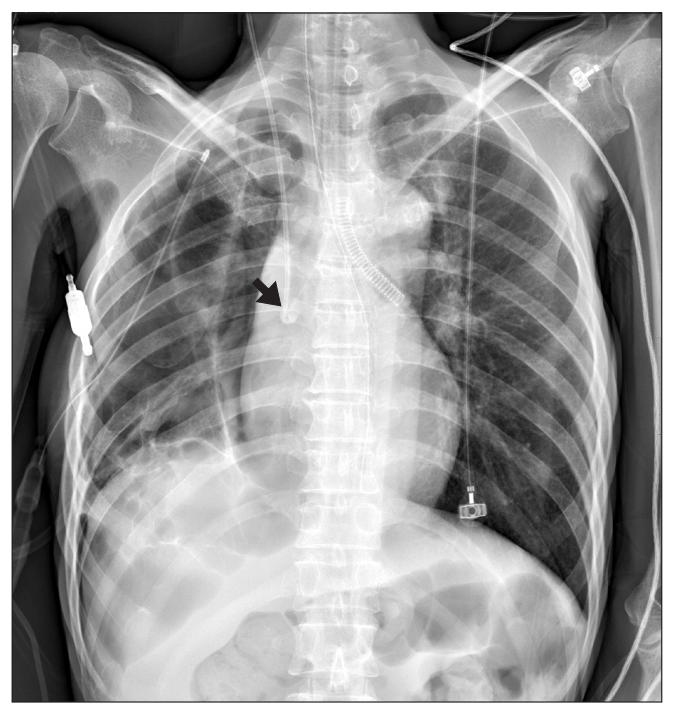

Fig. 2. The right lower lobe and middle lobe bronchi were blocked by the cuff (arrow).

a 35-French (Fr) DLT (Human Broncho, bronchial cuff OD 10.0 $\mathrm{mm}$, Insung Medical Co., Seoul, Korea), as the size of the patient's left main bronchus was $10.5 \mathrm{~mm}$ on CT, and the tube was fixed at a depth of $28 \mathrm{~cm}$ considering her height [2]. After fixing the DLT, we placed a bronchoscope (diameter $=3.1 \mathrm{~mm}$; Olympus LF-DP, Tokyo, Japan) on the tracheal lumen to confirm the position of the DLT, and then performed one-lung ventilation. We inserted a 4-Fr Fogarty Arterial Embolectomy Catheter (Edwards Lifesciences Co., Irvine, CA, USA) into the tracheal lumen and confirmed that it entered the right main bronchus via the bronchoscope and C-MAC monitor (Karl Storz GmbH \& Co. KG, Tuttlingen, Germany). We removed the stylet and connected a $3 \mathrm{ml}$ syringe filled with a contrast medium and normal saline to the 3-way stopcock. After passing through right upper lobe opening, we confirmed the right middle and lower lobes;

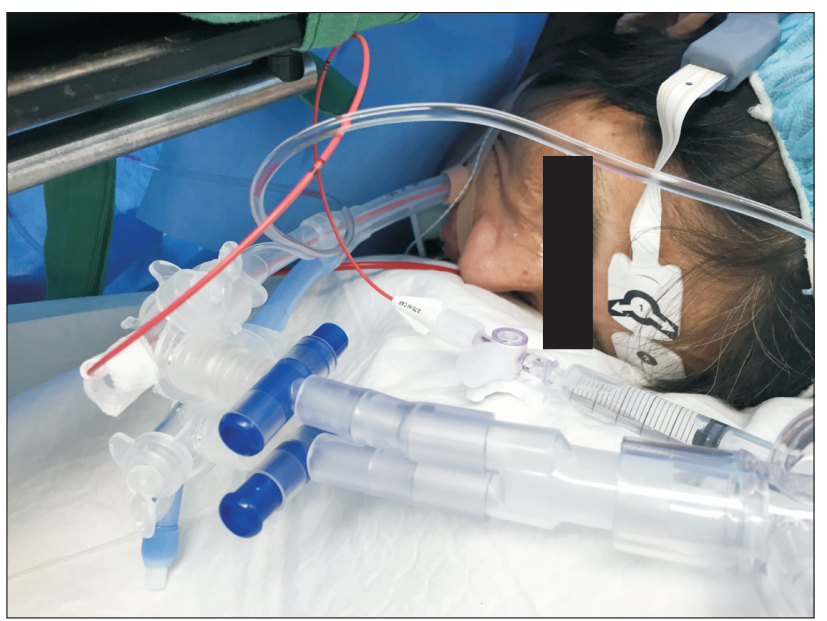

Fig. 3. A Fogarty catheter was fixed within a double-lumen tube with the patient in the lateral position.

we confirmed with a bronchoscope that the distance from the origin of the right upper lobe to the right middle and lower lobe bifurcation point was $3 \mathrm{~cm}$. After inserting the Fogarty catheter $3 \mathrm{~cm}$ further based on the distance that we measured, we performed cuff ballooning using $0.7 \mathrm{ml}$ of solution mixed with normal saline (Fig. 1). We confirmed that the cuff blocked the middle and lower lobe bifurcation on chest radiography (Fig. 2). Additionally, left radial artery cannulation and right internal jugular vein catheterization were performed.

Subsequently, we moved the patient to a lateral position while monitoring the movement of the Fogarty catheter via the bronchoscope (Fig. 3). After removing the contrast agent from the cuff, $1 \mathrm{ml}$ of air was inserted into the cuff to inflate the balloon. The cuff was maintained at a pressure less than $20 \mathrm{mmHg}$ using a Hi-LO ${ }^{\mathrm{TM}}$ Hand Pressure Gauge (Mallinckrodt Medical, Athlone, Ireland). The Fogarty catheter was removed after placing a surgical clamp on the bronchus of the right middle lobe. 
Postoperatively, the patient's tube was replaced with a $7.0-\mathrm{mm}$ single lumen tube, and she was transferred to the intensive care unit (ICU). Her oxygen saturation was maintained at 99-100\% during the operation, and chest radiographic findings did not show signs of pneumonia in the right upper lobe. Extubation was performed $48 \mathrm{~h}$ after surgery.

\section{Discussion}

When lobectomy is performed to treat a lung abscess, it is important to prevent the spread of infection to the non-affected lung and then prevent the infection from spreading to the remaining parts of the ipsilateral lung. These steps are especially important in cases in which the patient's general condition is weak due to recurrent pneumonia as well as impaired motor and cognitive function, which is typical in advanced stage Huntington's disease [3].

Selective lobar isolation prevents the endobronchial spread of infection, reduces postoperative complications, and improves the patient's oxygenation via either $\mathrm{O}_{2}$ flow or continuous positive airway pressure when hypoxia occurs during surgery $[4,5]$. This selective lobar isolation is performed by combining a bronchial blocker to the DLT or by using an uninvent tube. Although an uninvent tube is easy to manipulate, if the cuff is moved or damaged during surgical manipulation, it increases the risk of spreading the lung abscess. In addition, selective ventilation of the ipsilateral lobe is limited. In contrast, using a bronchial blocker with a DLT can protect the left lung even if the bronchial blocker is damaged during surgery. Blockers that can be used in connection with DLT include Arndt blockers and Fogarty embolectomy catheters, which are used for removal of a thrombus. Fogarty embolectomy catheters were preferred over Arndt blockers as they are more economical and are always available at our hospital. However, these blockers also have the following limitations: 1) they can be displaced even after fixation because of the patient's positioning and intraoperative movement; 2) they restrain the deflation of the lung after isolation, because there is no lumen or the lumen is very small within the catheter; and 3) they are not a viable option for patients with a latex allergy or stylet-induced damage of the bronchus [5-7].

We intermittently monitored our patient via the monitor connected to the bronchoscope to minimize movement of the catheter when she was in the lateral position. The right middle and lower lobes were isolated, which prevented lung collapse, but this did not limit the view during surgery. We removed the stylet after the catheter was inserted into the right main bronchus to prevent stylet-induced damage.

The maximal liquid and gas capacity of the balloon for 4-Fr size Fogarty embolectomy catheters are $0.7 \mathrm{ml}$ and $1.5 \mathrm{ml}$, respectively, and the size of the inflated balloon is $9 \mathrm{~mm}$. Based on these specifications, we determined that it is adequate for blocking the 6-6.5 $\mathrm{mm}$ intermediate bronchus as measured with CT. We performed ballooning with $0.7 \mathrm{ml}$ of contrast medium and confirmed that the intermediate bronchus was completely blocked. In addition, we verified on the chest radiograph that the balloon tip was placed at the bifurcation of the right middle and lower lobes. To prevent the cuff that was filled with contrast medium from rupturing and the lumen from being obstructed, we performed re-ballooning with $1 \mathrm{ml}$ of air.

We placed the patient in a $15^{\circ}$ head-up position after the patient arrived in the operating room and performed a rapid sequence induction without ventilation to minimize the spread of the abscess [8]. The patient's oxygen saturation decreased to 95\% during the process of confirming the final position of the DLT via the bronchoscope, but it improved after we commenced one-lung ventilation. Intraoperative arterial blood gas analysis confirmed that the patient's partial pressure of oxygen was maintained at $184 \mathrm{mmHg}$ with a fractional inspired $\mathrm{O}_{2}$ level of 1.0, so we did not administer an additional supply of $\mathrm{O}_{2}$ to the right upper lobe. Pathological testing following surgery confirmed that aspiration was the cause of the lung abscess. Extubation was performed on postoperative day 2 , as chest radiography in the ICU did not show abnormal findings.

The primary lesions in the present case were in the right middle lobe, which was suggestive of necrotizing pneumonia, and the right lower lobe, which was suggestive of aspiration pneumonia. Therefore, the primary objective was to block the point of division of the middle and lower lobes with a Fogarty catheter to prevent the abscess in the right middle lobe from contaminating the right lower and upper lobes (Fig. 4). However, one limitation is that an imperfect fit between the bronchus and the

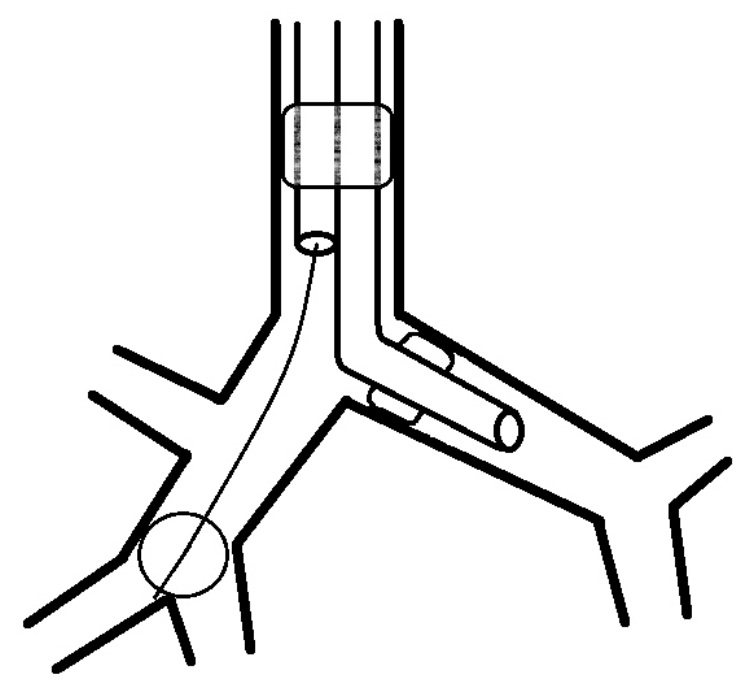

Fig. 4. Schematic diagram of the position of the balloon. 
cuff cannot completely isolate the right middle lobe; thus, the abscess can still spread from the middle lobe to the lower lobe, which would not be observed via the bronchoscope during surgery. Another limitation is that there is the possibility of the lung abscess spreading further during the process of removing the catheter and/or deflating the balloon. This risk can be reduced by adjusting the patient's position to a supine position. Selective isolation of the right middle lobe could have been an option; however, we did not perform right middle lobar isolation as the abscess may have spread to the lower or upper lobe during the insertion of a Fogarty catheter into the middle lobe. One of the typical symptoms of Huntington's disease is difficulty swallowing, and the resulting pneumonia is the most frequent cause of death [4]. Therefore, selective lobar isolation-an attempt to preserve the functional lobes during surgery-can be a good ventilation method during lobectomy for patients with Huntington's disease or an overall impairment of pulmonary function.

\section{ORCID}

Jaewon Kim, http://orcid.org/0000-0002-7256-6930

Chang-Young Jeong, http://orcid.org/0000-0003-2810-2511

\section{References}

1. Licker M, Le Guen M, Diaper J, Triponez F, Karenovics W. Isolation of the lung: double-lumen tubes and endobronchial blockers. Trends Anaesth Crit Care 2014; 4: 47-54.

2. Brodsky JB, Benumof JL, Ehrenwerth J, Ozaki GT. Depth of placement of left double-lumen endobronchial tubes. Anesth Analg 1991; 73: 570-2.

3. Roos RA. Huntington's disease: a clinical review. Orphanet J Rare Dis 2010; 5: 40.

4. McGlade DP, Slinger PD. The elective combined use of a double lumen tube and endobronchial blocker to provide selective lobar isolation for lung resection following contralateral lobectomy. Anesthesiology 2003; 99: 1021-2.

5. Vretzakis G, Dragoumanis C, Papaziogas B, Mikroulis D. Improved oxygenation during one-lung ventilation achieved with an embolectomy catheter acting as a selective lobar endobronchial blocker. J Cardiothorac Vasc Anesth 2005; 19: 270-2.

6. Campos JH. An update on bronchial blockers during lung separation techniques in adults. Anesth Analg 2003; 97: 1266-74.

7. Sharma A, Sinha S, Khanna S, Mehta Y, Khandelwal S, Khan AZ. A novel technique to prevent endobronchial spillage during video assisted thoracoscopic lobectomy. Ann Card Anaesth 2014; 17: 164-6.

8. Pfitzner J, Peacock MJ, Tsirgiotis E, Walkley IH. Lobectomy for cavitating lung abscess with haemoptysis: strategy for protecting the contralateral lung and also the non-involved lobe of the ipsilateral lung. Br J Anaesth 2000; 85: 791-4. 